\title{
COMPARATIVE STUDY OF FIELD DESORPTION AND SECONDARY ION MASS SPECTRA FOR ANTIBIOTICS
}

\author{
HIDEKI KAMBARA* \\ Central Research Laboratory, Hitachi Ltd., Kokubunji, Tokyo 185, Japan \\ ShinZaburo Hishida \\ Naka Works, Hitachi Ltd., Katsuta, Ibaragi 312, Japan \\ Hiroshi Naganawa \\ Institute of Microbial Chemistry, \\ Kamiosaki, Shinagawa-ku, Tokyo 141, Japan
}

(Received for publication October 9, 1981)

\begin{abstract}
Nonvolatile and thermolabile antibiotics are investigated both by field desorption and by secondary ion mass spectrometry (SIMS). It has been successfully demonstrated that these two methods are complementary in obtaining informations about molecular weight and structure. Although FD is most widely used for the investigation of nonvolatile biologically active compounds, there are some compounds of which FD can not provide reliable information. SIMS is successfully applied to obtain structural information of these compounds. SIMS spectra frequently depend on the surface conditions of sample holder materials.
\end{abstract}

There are many antibiotics thermally very labile and nonvolatile of which electron impact or chemical ionization cannot be used to obtain information on molecular weight and structure. Field desorption (FD) mass spectrometry has been successfully applied to these compounds ${ }^{1,2)}$. However, even FD has some drawbacks. It requires a special, high quality, ion emitter to obtain good FD spectra that are structurally informative. Precise temperature control of the emitter is also required for FD measurement. Furthermore, FD does not provide any information on molecular weight and structure for some compounds.

Recently, several new ionization methods that complement FD have been reported. One of them is secondary ion mass spectrometry (SIMS) ${ }^{3 \sim 5)}$. The term FAB (fast atom bombardment) is used when the primary particles are neutral atoms instead of ions ${ }^{\ell, 7}$. SIMS seems to be quite adequate for ionizing thermolabile compounds because it does not require heating. Many biologically active compounds have already been investigated by SIMS $^{8 \sim 10)}$.

The purpose of this paper is to compare FD and SIMS spectra for several antibiotics and to demonstrate that they are complementary in obtaining structural information. It is also demonstrated for several compounds that SIMS provides quasimolecular ions, which are hardly produced by FD.

\section{Experimental}

FD spectra were obtained using a Hitachi M-80 double focusing mass spectrometer and a Hitachi 003 data processing system. The ion acceleration energy was $3 \mathrm{keV}$ (a mass range from 1 to 1500 daltons can be covered at this acceleration energy). The extraction voltage supplied between the emitter and the counter electrode was $9 \mathrm{kV}$. Each sample was dissolved in water - methanol (1:4) at a ratio of $1 \mu \mathrm{g} / \mu \mathrm{l}$. An FD emitter having carbon microneedles on a thin tungsten wire $(10 \mu \phi)$ was used. After loading 
less than $1 \mu \mathrm{g}$ of each sample on to the emitter, FD spectra were repeatedly recorded and stored in the data processing system while increasing the emitter heating current at a constant rate $(2 \mathrm{~mA} / \mathrm{minute})$. All FD spectra obtained by repetitive scan were integrated to produce an average FD spectrum for each sample. No special technique, such as rapid heating of the emitter, was used in this experiment.

SIMS spectra were obtained with a double focusing mass spectrometer(Hitachi RMU 7D) modified for SIMS study. Details of the apparatus have been reported previously ${ }^{10)}$. The acceleration voltage was $1.3 \mathrm{kV}$ for samples smaller than 750 daltons and $1.2 \mathrm{kV}$ for lividomycin A (m.w. 761). Xenon ions were used as primary ions to bombard a $2 \mathrm{~mm} \times 8 \mathrm{~mm}$ silver substrate with an energy of $3.7 \mathrm{keV}$. The $\mathrm{Xe}^{+}$ion current was $1 \times 10^{-9} \mathrm{~A}$.

A half $\mu \mathrm{g}$ of each sample was loaded on the silver substrate and introduced to the bombardment source with a modified direct inlet probe. Primary ions hit the substrate at $45^{\circ}$ angle. Secondary ion mass spectra were recorded using an UV recorder.

\section{Results and Discussion}

Kasugamycin (m.w. 379)

Kasugamycin is an aminoglycoside having an amidine carboxyl group and thermally labile so that loss of the $\mathrm{CO}_{2}$ group from the molecule can easily occur even in FD ionization. An FD spectrum of kasugamycin is shown in Fig. 1-a. $\mathrm{MH}^{+}$is the base peak and fragment at $\mathrm{m} / \mathrm{z} 336$ is due to the loss of $\mathrm{CO}_{2}$ from $\mathrm{MH}^{+}$, which is the only fragment abundantly observed in the spectrum. The FD spectrum is not very informative about molecular structure.

Molecular ion species such as $(\mathrm{M}+2 \mathrm{Na}-\mathrm{H})^{+}$and $(\mathrm{M}+\mathrm{Ag})^{+}$are observed in the SIMS spectrum shown in Fig. 1-b. Many fragments, including $\left(\mathrm{M}-\mathrm{CO}_{2}+\mathrm{Na}\right)^{+}$and $\left(\mathrm{M}-\mathrm{CO}_{2}+\mathrm{Ag}\right)^{+}$, are observed. They are ion at $m / z 223$, due to a glycosidic bond cleavage, and ions at $m / z 231$ and 244, due to ring ruptures. These are very informative about the molecular structure. Cationized molecules $(\mathrm{M}+2 \mathrm{Na}-$ $\mathrm{H})^{+}$frequently appear in the SIMS spectrum of a compound having a $\mathrm{COOH}$ group when alkali metal salts, such as $\mathrm{NaCl}$, coexist in the sample. Argentated molecules are also frequently observed in SIMS spectra when silver is used as a substrate. These are very helpful in identifying molecular weight because of the $\mathrm{Ag}$ doublet (m.w.+107 and 109).

Lividomycin A (m.w. 761)

Lividomycin $\mathrm{A}$ is an aminoglycoside antibiotic with five saccharide units. Although the molecular species could be observed in the FD spectrum, there were many ions in the molecular ion region which could not be elucidated from the molecular structure. Many fragment ions were observed as well. However, most of them could not be elucidated from the molecular structure. Furthermore, the reproducibility of the spectrum was not good enough to obtain information about molecular weight and structure.

Fig. 1. FD and SIMS spectra of kasugamycin. (a) FD spectrum, (b) SIMS spectrum.
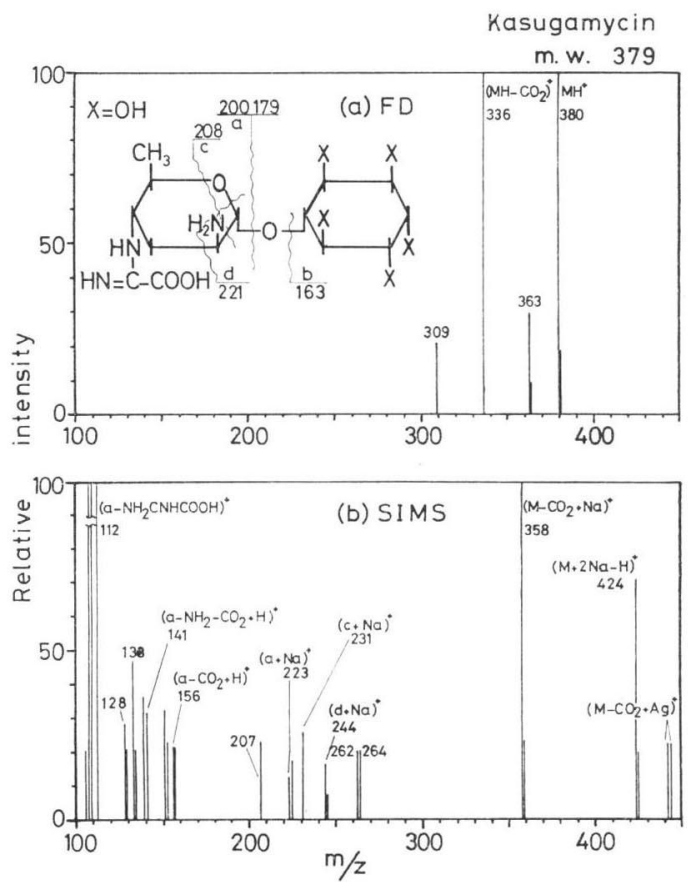
Fig. 2. SIMS spectrum of lividomycin A.

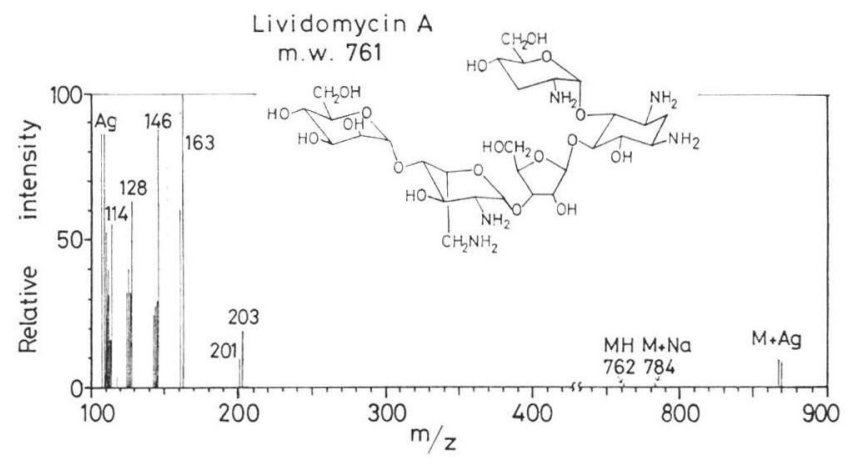

Fig. 3. SIMS spectrum of dihydrostreptomycin.

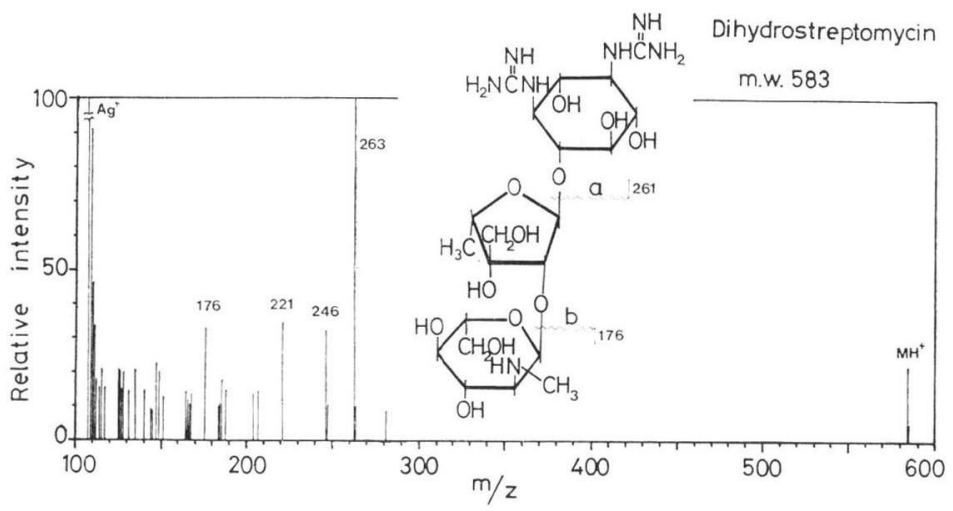

However, in the SIMS spectrum, argentated molecules $(\mathrm{M}+\mathrm{Ag})^{+}$are clearly observed at $\mathrm{m} / \mathrm{z} 868$ and 870 , as shown in Fig. 2. These peaks are shifted to $m / z 784$ by the addition of sodium chloride. Only small fragments are observed. The ions at $m / z 124$, and 163 are due to monosaccharides and their dissociation products.

Dihydrostreptomycin (m.w. 583)

Dihydrostreptomycin is a nonvolatile and thermolabile aminoglycoside having two guanidino groups. No molecular ion species or structurally informative fragments can be observed in its FD spectrum. However, quasimolecular ions $\mathrm{MH}^{+}$and argentated molecules $(\mathrm{M}+\mathrm{Ag})^{+}$are clearly and reproducibly observed in a SIMS spectrum, as shown in Fig. 3. The fragments at $m / z 263$ and 176 are due to bond ruptures at "a" and "b" respectively, as indicated in the figure. The same fragments are observed in a SIMS spectrum for streptomycin, which has a $\mathrm{CHO}$ group instead of a $\mathrm{CH}_{2} \mathrm{OH}$ group in the second saccharide ring. Ion species observed in the SIMS spectrum are quite reproducible, but their relative ion intensities changed $20 \sim 30 \%$ due to measurement conditions.

Minosaminomycin (m.w. 618)

Minosaminomycin has both peptide and aminoglycoside moieties. The quasimolecular ion $\mathrm{MH}^{+}$ and many fragments are observed in its FD spectrum, as shown in Fig. 4-a, The base peak is the ion at $m / z 294$ which also appears in the SIMS spectrum (Fig. 4-b). Fragments at $m / z 308(306+2 \mathrm{H}), 334,447$, 
Fig. 4. FD and SIMS spectra of minosaminomycin: (a) FD spectrum, (b) SIMS spectrum.

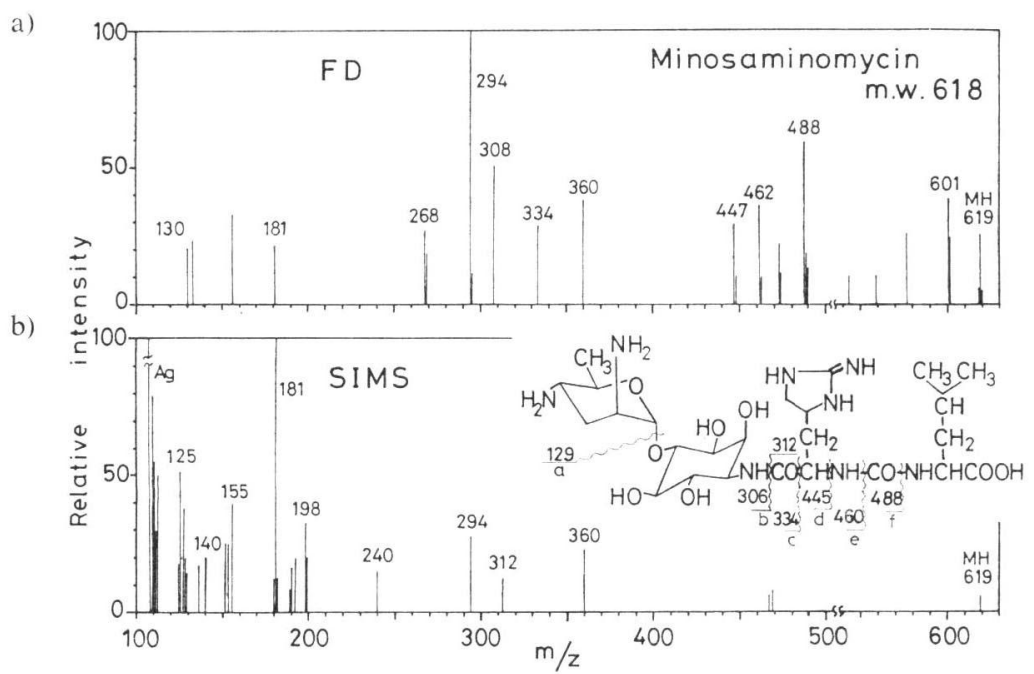

462, and 488 are produced by bond ruptures at "b", "c", "d", "e", and "f", respectively, as indicated in the figure. The fragment at $m / z 360$ is probably due to simultaneous bond ruptures at "a" and " $\mathrm{f}$ ", accompanied by one hydrogen attachment. The FD spectrum of minosaminomycin is very informative about molecular structure.

It is not easy to observe molecular ion species, such as $\mathrm{MH}^{+}$, in a SIMS spectrum. The molecular species $\mathrm{MH}^{+}$is observed only after adding a small amount of $\mathrm{NH}_{4} \mathrm{Cl}$ to the sample, or after adding a small amount of $\mathrm{NH}_{4} \mathrm{Cl}$ on the sample holder prior to sample loading. The favorable effects arising from coexisting $\mathrm{NH}_{4} \mathrm{Cl}$ in SIMS studies was first demonstrated by Cooks et al. for sucrose ${ }^{2)}$. Although the addition of $\mathrm{NH}_{4} \mathrm{Cl}$ was not necessary for oligosaccharides in our SIMS studies ${ }^{8,10)}$, molecular ion species of riboflavine or adenosinemonophosphate (AMP) are not easily observed without $\mathrm{NH}_{4} \mathrm{Cl}$ or $\mathrm{HCl}$ in positive ion mode. However, the presence of these does not always give good results for some other compounds. The effect of coexisting materials should be clarified for better understanding of ion formation processes in SIMS.

The SIMS spectrum in Fig. 4-b was obtained in the presence of $\mathrm{NH}_{4} \mathrm{Cl}$. The base peak is the ion at $m / z$ 181, which include enduracididine. The fragments at $m / z 198,294\left(312-\mathrm{H}_{2} \mathrm{O}\right)$, and 312 are due to bond cleavages, as shown in the figure. They all include enduracididine, which indicates that this amino acid is easily ionized and stable as an ion. Analogous fragments, including a cyclic guanidino moiety which is very stable as an ion, are frequently observed in SIMS spectra (see also the SIMS spectrum for chymostatin below). Generally, these species can not be observed in an FD spectrum. Consequently, a SIMS spectrum complements an FD spectrum.

\section{Phosphoramidon (m.w. 543)}

Phosphoramidon contains a saccharide part connected to a peptide part through a phosphate bond. Molecular ion species are not observed in its FD spectrum, as shown in Fig. 5-a. Only fragments at $m / z$ 299 and 317 are abundant. These are due to the peptide moiety.

The observation of molecular species is not easy in its SIMS spectrum, either, without coexisting salts. The sodiated molecules $(\mathrm{M}+2 \mathrm{Na}-\mathrm{H})^{+}$are clearly observed in the SIMS spectrum after adding 
Fig. 5. FD and SIMS spectra of phosphoramidon: (a) FD spectrum, (b) SIMS spectrum.

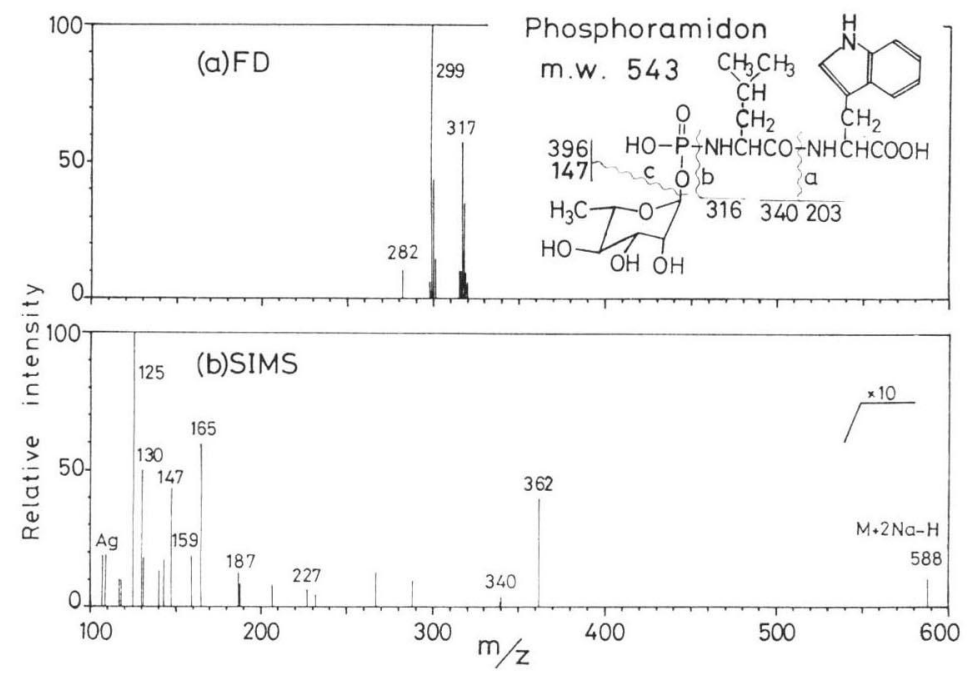

Fig. 6. FD and SIMS spectra of blasticidin S: (a) FD spectrum, (b) SIMS spectrum.

a)

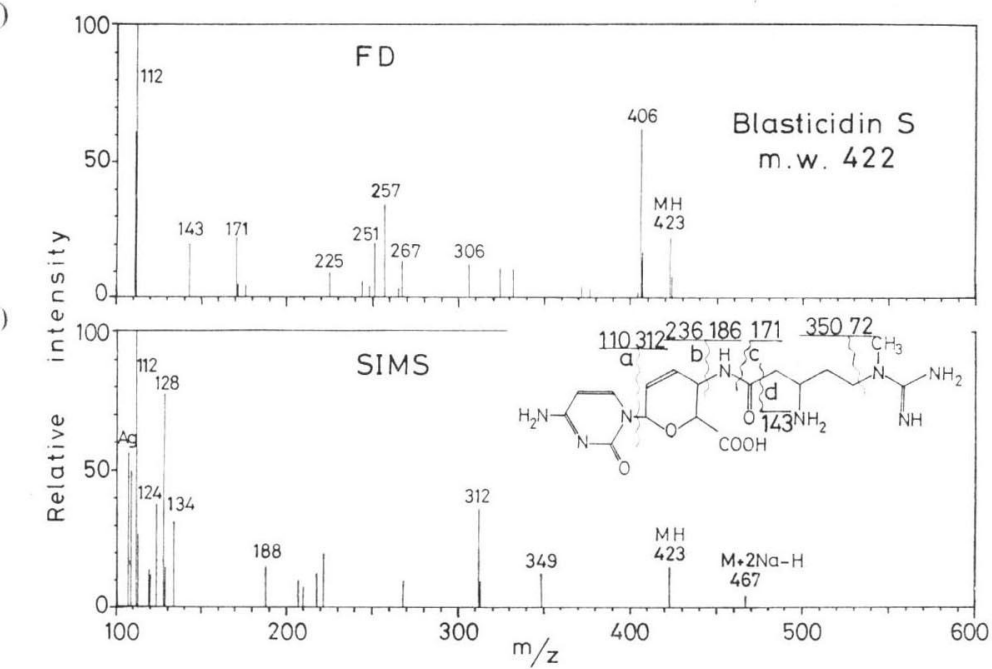

a small amount of sodium chloride on the silver surface before sample loading (Fig. 5-b). Many structurally informative fragments appear. The fragments at $\mathrm{m} / z 130$ and 159 are due to amino acid moieties and the fragments at $\mathrm{m} / \mathrm{z} 147$ and 165 are due to the saccharide moiety. It may be reasonable to assume that the fragments at $\mathrm{m} / \mathrm{z} 362$ are produced by a bond cleavage at "b" accompanied by two sodium attachments.

Blasticidin S (m.w. 422)

Blasticidin S has very polar groups, such as amino, carboxyl, and guanidino groups. Quasimolecular ions $\mathrm{MH}^{+}$are observed in its FD spectrum, as shown in Fig. 6-a. The base peak is the ions at $m / z$ 112 which is due to cytosine. Most of the fragments can be elucidated from the molecular structure. The fragments at $m / z 143$ and 171 are due to bond cleavages at " $d$ " and "c", respectively. The ion at $m / z 406$ must be $\left(\mathrm{MH}^{+}-\mathrm{NH}_{3}\right)$. The ion structures at $m / z 257$ and 306 are not clear. 
Fig. 7. SIMS spectrum of chymostatin.
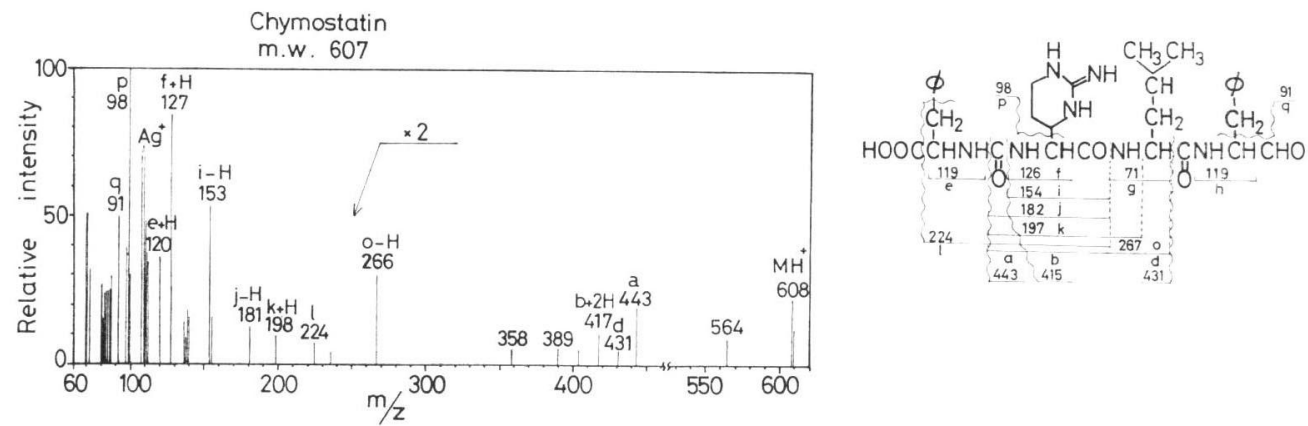

Fig. 8. SIMS spectrum of antipain

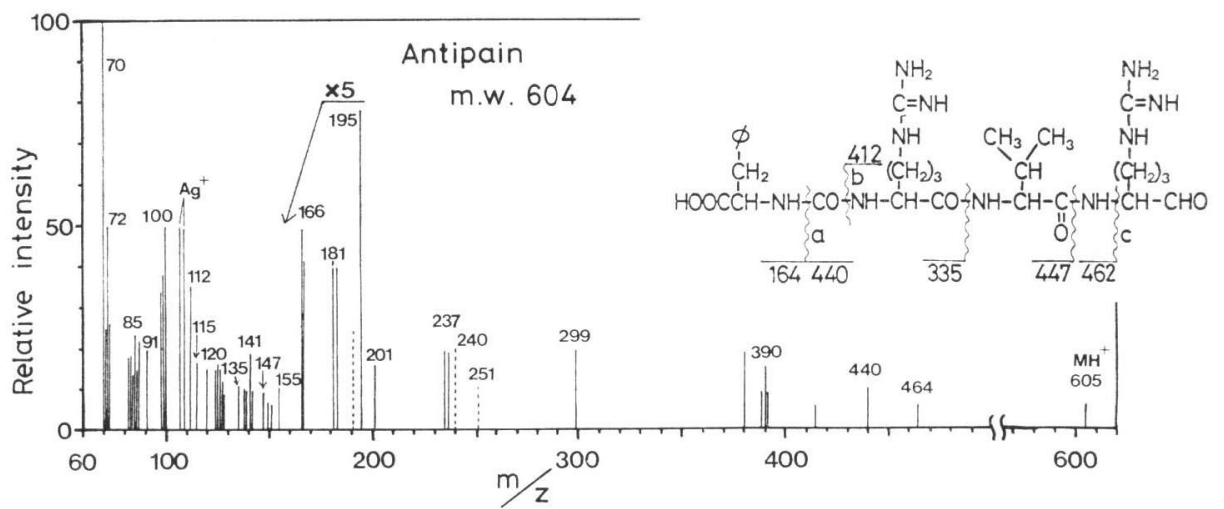

Quasimolecular ions $\mathrm{MH}^{+}$are observed in the SIMS spectrum, but are not reproducible without coexisting salts. The quasimolecular ions are reproducibly observed after adding a small amount of ammonium chloride on the silver substrate before sample loading. The fragments at $\mathrm{m} / \mathrm{z} 112$ and 312 are due to bond cleavage at "a". The former also appears in the FD spectrum, but the latter does not. Some of the fragments observed in the SIMS spectrum differ from those observed in the FD spectrum. Therefore they provide complementary information about the molecular structure.

\section{Chymostatin (m.w. 607)}

Chymostatin, an inhibitor for chymotrypsin, has a very polar amino acid, cyclic guanidino amino acid. Therefore, it is very difficult to observe molecular ion species in its mass spectrum. Actually, neither the molecular species nor any structurally informative fragments can be observed even in the FD spectrum. However, quasimolecular ions, together with a lot of structurally informative fragments, appear in the SIMS spectrum, as shown in Fig. 7. The fragments at $m / z$ 72, 120 and 127 are due to amino moieties. The ions at $m / z 153,198$, and 267 must be fragments including the cyclic guanidino moiety. These fragments are produced by the bond cleavages indicated in the figure. The fragments at $m / z 443,415$ and 431 are due to bond cleavages at "a", "b" and "d", respectively.

Antipain (m.w. 604)

Antipain, an inhibitor of papain, also has very polar groups, such as guanidino, carboxyl, and formyl groups, and therefore is very thermally labile and nonvolatile. Neither molecular ion species nor 
structurally informative fragments could be observed in the FD spectrum obtained in this study.

It is not easy to observe molecular ion species in a SIMS spectrum, either. A SIMS spectrum depends strongly on surface conditions. Good results were not obtained with a used silver substrate, or with a silver substrate treated in a hydrogen flame for a long period. The SIMS spectrum shown in Fig. 8 was finally obtained using a new silver substrate treated in a hydrogen flame for a short period. Quasimolecular ions $\mathrm{MH}^{+}$are observed. The dashed lines indicate that observation of those species is not reproducible. The fragments at $m / z 440$ are produced by a bond cleavage at " $\mathrm{a}$ ". The fragments at $m / z$ 166, 414, and 464 are due to bond ruptures at "a", "b", and "c" accompanied by two hydrogen attachments. The fragments at $\mathrm{m} / \mathrm{z} 72,91,100$ and 120 must be due to amino moieties in the compound.

\section{Conclusion}

It has been successfully demonstrated that SIMS can provide information about molecular weight and structure for thermolabile and nonvolatile antibiotics, some of which cannot be obtained from FD spectra. In most cases, the information from SIMS spectra complements that from FD spectra. There are several compounds in which the SIMS spectra are strongly influenced by surface conditions of the substrate. They especially depend on coexisting materials on the surface. The study of the effects caused by coexisting materials is most important for a better understanding of SIMS and its ionization processes. Consequently, such study is necessary for improvement in SIMS sensitivity and reproducibility.

\section{References}

1) Beckey, H. D.: Principles of Field Ionization and Field Desorption Mass Spectrometry, Pergamon, Oxford, 1977

2) Schulten, H. R.: Biochemical, medical, and environmental applications of field ionization and field desorption mass spectrometry. Int. J. Mass Spectrom. Ion Phys. 32: 97 283, 1979

3) Benninghoven, A.; D. Jaspers \& W. Sichtermann: Secondary ion emission of amino acids. Appl. Phys. 11: 35 39, 1979

4) Benninghoven, A. \& W. K. Sichtermann: Detection, identification and structural investigation of biologically important compounds by secondary ion mass spectrometry. Anal. Chem. 50: 1180 1184, 1978

5) DAY, R. J.; S. E. Unger \& R. G. COOKs: Molecular secondary ion mass spectrometry. Anal. Chem. 52: 557A $\sim 572 \mathrm{~A}, 1980$

6) Surman, D. J. \& J. C. Vickerman: Fast atom bombardment quadrupole mass spectrometry. J. C. S. Chem. Comm. 1981: 324 325, 1981

7) Barber, M.; R. S. Bordoli, R. D. Sedgwick \& A. N. Tyler: Fast atom bombardment of solids (F. A. B.): A new ion source for mass spectrometry. J. C. S. Chem. Comm. 1981: 325 327, 1981

8) Kambara, H. \& S. Hishida: Secondary ion mass spectra of oligosaccharides. Org. Mass Spectrom. 16: $167 \sim 170,1981$

9) Liu, L. K.; K. L. Busch \& R. G. Cooks: Matrix assisted secondary ion mass spectra of biological compounds. Anal. Chem. 53: 109 113, 1981

10) Kambara, H. \& S. Hishida: Secondary ion mass spectra of nonvolatile bio-organic compounds. Anal. Chem. 53: Dec. issue, 1981 